\title{
EVALUATION OF CHANGES IN VISUAL ACUITY AND CONTRAST SENSITIVITY IN PATIENTS WITH KERATOCONUS AFTER CORNEAL COLLAGEN CROSS-LINKING
}

\author{
Hassaan Javaid, Omar Zafar, Seemal Akram*, Asfandyar Khan, Sana Abbas, Aisha Rafique \\ Armed Forces Institute of Ophthalmology/National University of Medical Sciences (NUMS) Rawalpindi Pakistan, *PAED General Hospital, Islamabad Pakistan
}

\section{ABSTRACT}

Objective: To evaluate changes in visual acuity and contrast sensitivity in patients with keratoconus after corneal collagen cross-linking.

Study Design: Quasi-experimental study.

Place and Duration of Study: Armed Forces Institute of Ophthalmology, Rawalpindi, Pakistan, from May 2019 to Apr 2020. Methodology: A total of 48 eyes of 30 patients with progressive keratoconus were included in this study. Baseline uncorrected distance visual acuity, corrected distance visual acuity and contrast sensitivity were recorded, following which transepithelial corneal collagen cross-linking with topical riboflavin and ultraviolet A light was performed. Uncorrected distance visual acuity, corrected distance visual acuity and contrast sensitivity measurements were repeated and recorded 6-months postoperatively.

Results: Mean preoperative uncorrected distance visual acuity was $0.56 \pm 0.27$ logarithm of the minimum angle of resolution which improved to $0.51 \pm 0.26$ at 6 months after the procedure $(p=0.002)$. Mean corrected distance visual acuity was $0.30 \pm 0.19$ preoperatively, improving to $0.24 \pm 0.18(p=0.001)$ at 6 months after corneal collagen cross-linking, thereby depicting a statistically significant improvement. Treated eyes also showed a significant improvement in contrast sensitivity $(p=0.001)$ of $0.05 \pm$ 0.08 logarithmic units of contrast sensitivity, from a mean preoperative contrast sensitivity of $1.72 \pm 0.10$ to $1.77 \pm 0.09$ when evaluated 6 months after corneal collagen cross-linking.

Conclusion: Corneal collagen cross-linking is a promising advancement in the treatment of keratoconus. It was found effective in significantly improving visual acuity as well as contrast sensitivity, thus enhancing visual outcomes in keratoconus.

Keywords: Corneal cross-linking, Contrast sensitivity, Keratoconus.

How to Cite This Article: Javaid H, Zafar O, Akram S, Khan A, Abbas S, Rafique A. Evaluation of Changes in Visual Acuity and Contrast Sensitivity in Patients with Keratoconus After Corneal Collagen Cross-Linking. Pak Armed Forces Med J 2021; 71(5): 1705-1708. doi: https://doi.org/10.51253/pafmj.v71i5.4360

This is an Open Access article distributed under the terms of the Creative Commons Attribution License (https://creativecommons.org/licenses/by-nc/4.0/), which permits unrestricted use, distribution, and reproduction in any medium, provided the original work is properly cited.

\section{INTRODUCTION}

Keratoconus $(\mathrm{KC})$ derived is from the Greek words 'Kéras' meaning cornea and 'Cōnus' meaning cone. ${ }^{1}$ It may be defined as a progressive non-inflammatory condition that is characterized by central thinning and bulging of the cornea resulting in cone-like protrusion of the corneal surface. ${ }^{2}$ While the reasons are not fully understood, the disease is thought to result from a blend of hereditary, natural and hormonal factors. $\mathrm{KC}$ has an estimated incidence of around 1 in every 2,000 persons. However, different studies report varying rates in different ethnic groups. Multiple epidemiological studies have reported people of Indian, Pakistani and Arab origins to make up a significantly greater percentage of patients with KC. ${ }^{3}$ Generally manifesting in late youth to early adulthood, several risk factors for the development of KC have also been described, which include atopy, vernal keratoconjunctivitis, excessive contact lens wear, positive family history

Correspondence: Dr Hassaan Javaid, Resident Ophthalmology, Armed Forces Institute Ophthalmology, Rawalpindi Pakistan

Received: 29 May 2020; revision received: 14 Jul 2020; accepted: 16 Jul 2020 and male gender. ${ }^{4}$

$\mathrm{KC}$ is usually bilateral but often asymmetric. The condition progressively weakens the cornea by reducing the number of corneal collagen cross-links causing the cornea to assume a deformed conical shape and in severe cases may cause marked scarring of the cornea. ${ }^{5}$ These structural changes bring about blurring of vision, diplopia, photosensitivity and are often accompanied by marked deterioration of visual acuity (VA) and contrast sensitivity (CS) if left untreated. ${ }^{6}$

Diagnosis of manifest KC can be made easily on slit-lamp examination and assessment of corneal curvature readings. Computerized corneal topography is a helpful tool, not only for the identification KC at an early stage, but also for monitoring disease progression. ${ }^{7,8}$

Treatment for $\mathrm{KC}$ is tailored according to the stage of the disease. In the initial stages, prescription eyeglasses prove helpful. As the condition progresses, special, rigid contact lenses may be prescribed to correct irregular corneal astigmatism. Intracorneal ring segment implantation is another treatment option in 
patients with clear cornea. However, in cases where significant scarring of the cornea ensues, corneal transplantation is needed. ${ }^{9}$

Corneal collagen cross-linking (CXL) is a treatment modality for $\mathrm{KC}$ that induces the development of covalent cross-links between corneal collagen fibrils. This is achieved by utilizing a photosensitive material, riboflavin, activated by ultraviolet irradiation of specific frequency and wavelength, thus enhancing the biomechanical stability of cornea. ${ }^{10}$

Rationale of our research was to evaluate impact of CXL on VA and CS in patients with progressive KC in terms of establishment of treatment significance.

\section{METHODOLOGY}

This study was conducted at Armed Forces Institute of Ophthalmology, Rawalpindi, Pakistan from May 2019 to April 2020 after due approval from the hospital ethical review committee vide certificate reference number 219/ERC/AFIO. A total of 48 eyes of 30 individuals diagnosed with progressive $\mathrm{KC}$, eligible for CXL treatment were enrolled in this quasi-experimental study using non-probability consecutive sampling. Sample size of 35 was calculated using OpenEpi sample size calculator keeping $95 \%$ confidence interval and anticipated population prevalence of $2.3 \% .11 \mathrm{~A}$ total of 48 eyes were included in the study to further reduce chances of bias and increase authenticity of the study.

Inclusion Criteria: Progressive KC in at least one eye, thinnest corneal pachymetry of more than or equal to $400 \mu \mathrm{m}$ in the eye undergoing CXL, absence of central corneal opacity and corrected distance visual acuity of 1.0 logarithm of the minimum angle of resolution (logMAR), equivalent to Snellen VA of $6 / 60$, or better. Eyes showing an increase in the steepest simulated keratometric reading of at least 1 diopter (D), measured through computerized videoke-ratography, or an increase in corneal astigmatism of $>1 D$ over the last 1 year were said to have progression.

Exclusion Criteria: Patients who had history of other ocular surface disea-ses, previous hydrops, systemic associations of KC like Down syndrome, autoimmune disease, pregnancy, lactation, active ocular infection or inflammation and those who had received CXL treatment or refractive surgery in the past. Corneas with preoperative thinnest pachymetry of $<400 \mu \mathrm{m}$ were not treated.

Informed consent was acquired from all patients and also from their guardians in case of patients less than 18 years of age. All patients had a complete ophthalmic examination including uncorrected distance visual acuity (UDVA), corrected distance visual acuity (CDVA), CS testing, slit lamp biomicroscopy, dilated fundus examination, corneal topography and pachymetry (Galilei G4) preoperatively and on post-operative follow-up visits at 3 and 6 months. Use of soft contact lenses was advised to be discontinued at 1 week prior and gas permeable lenses 2 weeks prior to baseline evaluation.

All patients included in our study underwent transepithelial CXL under topical anaesthesia. Transepithe-zlial riboflavin solution Peschke TE $(0.25 \%$ riboflavin, $1.2 \%$ HPMC, $0.01 \%$ benzalkonium chloride) was used, which promotes the penetration of riboflavin through intact epithelium. One drop of isotonic riboflavin solution was instilled every 2 minutes, continued for 30 minutes. Corneal thickness was reconfirmed to be $>400 \mu \mathrm{m}$ with ultrasound pachymeter (REICHERT iPac PACHYMETER) and riboflavin flare in the anterior chamber was confirmed on slit lamp examination using cobalt blue filter. The cornea was then exposed to ultraviolet-A (UV-A) light of $365 \mu \mathrm{m}$ wavelength at an irradiance of $9.0 \mathrm{~mW} / \mathrm{cm} 2$ for 10 minutes using CCL-365 VARIO. Distance of UV light source was kept around $5 \mathrm{~cm}$ from the corneal apex. A soft bandage contact lens was applied upon completion of the procedure and removed 7 days later. Patients were advised to use topical $0.5 \%$ moxifloxacin and $0.1 \%$ prednisolone acetate along with lubricant eye drops. Steroid eye drops were discontinued after 5 days.

Visual acuity and contrast sensitivity testing was done using computerized vision testing system (M\&S Smart System 20/20). Monocular UDVA and CDVA were recorded preoperatively and 6 months after CXL treatment using Snellen VA notation, and the measurements were converted to $\log M A R$ equivalents. For contrast sensitivity function, each eye was again tested separately. Patients wore trial frames and optimal refractive correction was given. Letter contrast test was used with chart luminance of $85 \mathrm{~cd} / \mathrm{m}^{2}$ for recording CS preoperatively and then at 6 months post-CXL. The test results were converted to logarithmic units of contrast sensitivity $(\log C S)$. SPSS-23 was used for entry and statistical analysis of data. Continuous variables such as age, UCVA, CCDVA and CS were described in terms of mean \pm standard deviation. Variances between the pretest-posttest groups were determined using the paired sample t-test. The $p$-value of $\leq 0.05$ was considered to be statistically significant. 


\section{RESULTS}

In total, 48 eyes of 30 patients with progressive KC were enrolled in the study and followed up for 6 months after receiving CXL treatment. Sixteen of the participants were male while 14 were female. Mean age of the participants was $17.43 \pm 5.72$ years (range: 8 32 years).

A statistically significant improvement was seen in both UCVA and CDVA after CXL treatment was administered. Mean preoperative UCVA was $0.56 \pm$ $0.27 \log$ MAR which improved to $0.51 \pm 0.26 \log$ MAR at 6 months after the procedure $(p=0.002)$. Mean CDVA was $0.30 \pm 0.19 \log$ MAR preoperatively, improving to $0.24 \pm 0.18 \operatorname{logMAR}(p=0.001)$ at 6 months after CXL, thereby depicting a statistically significant improvement.

The mean preoperative CS of all eyes enrolled in the study was $1.72 \pm 0.10 \log$ CS. This increased to a mean of $1.77 \pm 0.09 \log C S$ when evaluated 6 months after CXL (Figure-1). The mean improvement in CS comparing preoperative and 6 months postoperative values was $0.05 \pm 0.08(p=0.001)$ which was statistically significant (Table). Figure-2 showed the changes obser-

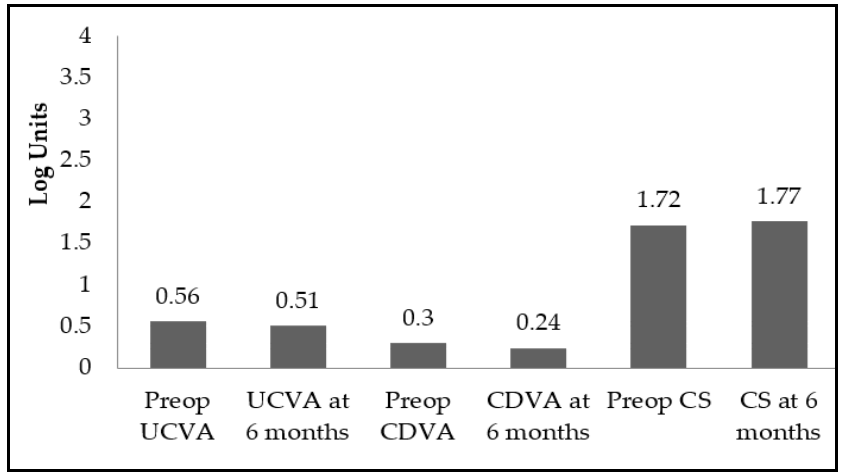

Figure-1: Preoperative and postoperative uncorrected distance visual acuity, corrected distance visual acuity and contrast sensitivity.

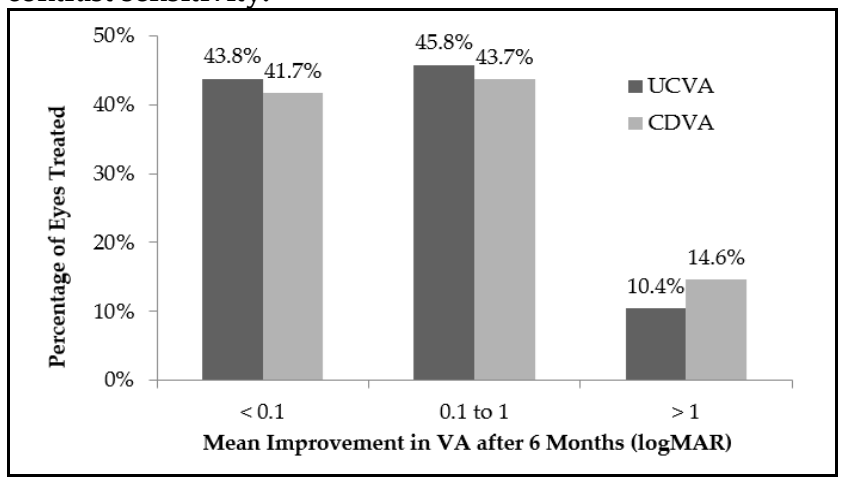

Figure-2: Percentage distribution of improvement in uncorrected distance visual acuity and corrected distance visual acuity 6 months after corneal collagen cross-linking ved in UCVA, CDVA and CS before and after CXL and demonstrate the significant difference observed at 6 months after treatment.

Table: Preoperative and postoperative uncorrected distance visual acuity, corrected distance visual acuity and contrast sensitivity.

\begin{tabular}{|c|c|c|c|}
\hline Study Parameters & $\begin{array}{l}\text { Mean } \pm \\
\text { SD }\end{array}$ & \begin{tabular}{|c|} 
Mean \\
Improvement \\
\end{tabular} & $\begin{array}{c}p \text { - } \\
\text { value }\end{array}$ \\
\hline $\begin{array}{l}\text { Preoperative uncorrected } \\
\text { distance visual acuity }\end{array}$ & $\begin{array}{c}0.56 \pm \\
0.27\end{array}$ & \multirow{2}{*}{$0.05 \pm 0.10$} & \multirow{2}{*}{$0.002 *$} \\
\hline $\begin{array}{l}\text { Uncorrected distance } \\
\text { visual acuity at } 6 \text { months }\end{array}$ & $\begin{array}{l}0.51 \pm \\
0.26\end{array}$ & & \\
\hline $\begin{array}{l}\text { Preoperative corrected } \\
\text { distance visual acuity }\end{array}$ & $\begin{array}{c}0.30 \pm \\
0.19\end{array}$ & \multirow{2}{*}{$0.06 \pm 0.10$} & \multirow{2}{*}{$0.001^{*}$} \\
\hline $\begin{array}{l}\text { Corrected distance visual } \\
\text { acuity at } 6 \text { months }\end{array}$ & $\begin{array}{c}0.24 \pm \\
0.18\end{array}$ & & \\
\hline $\begin{array}{l}\text { Preoperative contrast } \\
\text { sensitivity }\end{array}$ & $\begin{array}{c}1.72 \pm \\
0.10\end{array}$ & \multirow{2}{*}{$0.05 \pm 0.08$} & \multirow{2}{*}{$0.001^{*}$} \\
\hline $\begin{array}{l}\text { Contrast sensitivity at } 6 \\
\text { months }\end{array}$ & $\begin{array}{l}1.77 \pm \\
0.09\end{array}$ & & \\
\hline
\end{tabular}

\section{DISCUSSION}

CXL with riboflavin and UV-A light is a wellestablished treatment modality for progressive $\mathrm{KC}$ and corneal ectasias. The procedure results in significant increase in biomechanical rigidity of the cornea, thus checking disease progression. Although the primary objective of the procedure is to stabilize or halt progression of the disease, improvement in visual acuity has been reported in keratoconic eyes that underwent CXL. ${ }^{12}$ The distorted optical architecture of the cornea is primarily responsible for reduced vision in $\mathrm{KC}$. Improvement of corneal topography with $\mathrm{CXL}$ is therefore anticipated to be accompanied by improved visual function. This phenomenon may also be used as an indicator of CXL efficacy.

Hersh et al,13 reported an improvement of 5.7 letters of VA in keratoconic eyes at 12 months after CXL. There was a gain of approximately 2.2 letters in the control group showing a statistically significant difference in CDVA change at 1 year between the two groups $(p<0.01)$.

A meta-analysis of randomized control trials conducted by Li et al,14 showed that eyes treated with CXL had significantly improved CDVA in comparison with the control group $(p<0.001)$. However, UCVA was not significantly different in treatment and control groups ( $p=0.105)$.

Akbar et al,15 compared transepithelial CXL to epithelium-off CXL in the treatment of progressive KC in adult Pakistani population with regards to safety 
and efficacy of treatment. The study reports significant improvement in postoperative UCVA as well as CDVA at 12 months with the difference between the two groups being statistically insignificant $(p=0.650$ and 0.367 respectively). However, CDVA was significantly better in the transepithelial CXL group when assessed at 18 months $(p=0.036)$. Results generated from our study also depict that effective transepithelial CXL can bring about notable improvement in uncorrected as well as best spectacle corrected visual acuity.

CS is an important indicator of an individual's visual capability. Even with normal or near normal corrected visual acuity, CS in patients with $\mathrm{KC}$ is often suboptimal resulting in poor quality of vision. ${ }^{16}$ This aspect of visual assessment is often overlooked. Few studies have however reported that CS function improves following CXL.

In a study conducted by Zarei-Ghanavati et al,17 CS was found to decrease significantly at month 1 post-CXL. However, a rise in CS was seen when eyes were evaluated at 3,6 , and 8 months postoperatively. This improvement in contrast sensitivity after CXL was attributed to enhancement of corneal biomechanical stability along with reduction in the corneal curvature and astigmatism.

Lamy et al, ${ }^{18}$ studied 68 keratoconic eyes and reported improved CS in eyes that received CXL treatment after 2 years of follow-up $(p<0.001)$. No significant change in CS was reported by the study in control eyes over the same time period $(p=0.228)$. The mean improvement in CS measurements in eyes undergoing CXL was reported to be $0.16 \pm 0.12 \log C S$ at 2 years. This was higher than the mean change in CS of $0.05 \pm$ $0.08 \log C S$ seen after 6 months of the procedure in our study.

\section{LIMITATION OF STUDY}

The major limitations of our study were small sample size, lack of a control group and a relatively short follow-up period. Moreover, complications of CXL like corneal haze, infectious and diffuse lamellar keratitis, endothelial damage, stromal scarring and herpes virus reactivation were not described in our study. Larger studies with longer follow-up periods are required to better define the effects of CXL on visual outcomes in keratoconic eyes.

\section{CONCLUSION}

CXL is a promising treatment modality for progressive KC. It is an effective procedure which results in significant improvement in visual acuity as well as contrast sensitivity, thus enhancing visual outcomes. This technique may alle- viate morbidity besides halting keratoconic progression and avert the need for corneal transplantation.

\section{Conflict of Interest: None.}

\section{Authors' Contribution}

HJ: Conception, data collection, data analysis and manuscript writing, OZ: Research supervison and final approval, SA: Manuscritp editing, AK: Conception and research analysis, SA: Statistical analysis and interpretation, AR: Data collection.

\section{REFERENCES}

1. Kanellopoulos AJ, Moustou V, Asimellis G. Evaluation of visual acuity, pachymetry and anterior-surface irregularity in kerato-conus and crosslinking intervention follow-up in 737 cases. J Kerat Ect Cor Dis 2013; 2(3): 95-103.

2. Moghadam RS, Akbari M, Alizadeh Y, Medghalchi A, Dalvandi R. The outcome of corneal collagen cross-linking in patients with advanced progressive keratoconus: a 2-year follow-up study. Middle East Afr J Ophthalmol 2019; 26(1): 11-16.

3. Georgiou T, Funnell CL, Cassels-Brown A, O'Conor R. Influence of ethnic origin on the incidence of keratoconus and associated atopic disease in Asians and white patients. Eye (Lond) 2004; 18(4): 379-383.

4. Assiri AA, Yousuf BI, Quantock AJ, Murphy PJ. Incidence and severity of keratoconus in Asir province, Saudi Arabia. Br J Ophthalmol 2005; 89(11): 1403-1406.

5. Godefrooij DA, de Wit GA, Uiterwaal CS, Imhof SM, Wisse RP. Agespecific incidence and prevalence of keratoconus: a nation-wide registration study. Am J Ophthalmol 2017; 17(5): 169-172.

6. Srivastava G, Bhatia HK, Sridhar U. A study to evaluate the safety and efficacy of corneal collagen cross-linking with ribofla-vin in keratoconus patients. Indian J Clin Exp Ophthalmol 2018; 4(3): 333-338.

7. Cozma I, Atherley C, James NJ. Influence of ethnic origin on the incidence of keratoconus and associated atopic disease in Asian and white patients. Eye (Lond) 2005; 19(8): 924-926.

8. Kok YO, Tan GF, Loon SC. Review: keratoconus in Asia. Cornea 2012; 31(5): 581-593.

9. Kuechler SJ, Tappeiner C, Epstein D, Frueh BE. Keratoconus progression after corneal cross-linking in eyes with preoperative maximum keratometry values of 58 diopters and steeper. Cornea 2018; 37(11): 1444-1448.

10. Hersh PS, Lai MJ, Gelles JD, Lesniak SP. Transepithelial corneal cross linking for keratoconus. J Cataract Refract Surg 2018; 44(3): 313-322.

11. Gokhale NS. Epidemiology of keratoconus. Ind J Ophthalmol 2013; 61(8): 382-383.

12. Kaiserman I, Mimouni M, Rabina G. Epithelial photorefractive keratectomy and corneal cross-linking for keratoconus: the telaviv protocol. J Refract Surg 2019; 35(6): 377-382.

13. Hersh PS, Stulting RD, Muller D, Durrie DS, Rajpal RK, Binder PS, et al. United States multicenter clinical trial of corneal colla-gen crosslinking for keratoconus treatment. Ophthalmol 2017; 124(9): 1259-1270.

14. Li J, Ji P, Lin X. Efficacy of corneal collagen cross-linking for treatment of keratoconus: a meta-analysis of randomized cont-rolled trials. PLoS One 2015; 10(5): e0127079.

15. Akbar B, Ul-Haq RI, Ishaq M, Fawad A, Arzoo S, Siddidque K. Comparison of transepithelial corneal crosslinking with epithe-lium-off crosslinking (epithelium-off $\mathrm{CXL}$ ) in adult Pakistani population with progressive keratoconus. Taiwan J Ophthalmol 2017; 7(4): 185-190.

16. Bilen NB, Hepsen IF, Arce CG. Correlation between visual func-tion and refractive, topographic, pachymetric and aberrometric data in eyes with keratoconus. Int J Ophthalmol 2016; 9(8): 1127-1133.

17. Zarei-Ghanavati S, Khakshour H, Vejdani M, Ghooshkhanei H, Vejdani A. Evaluation of changes in visual acuity, contrast sensi-tivity and aberrations in patients with keratoconus after corneal collagen cross-linking. J Ophthalmic Vis Res 2017; 12(3): 260-264.

18. Lamy R, Netto CF, Reis RG, Procopio B, Porco TC, Stewart JM,. Effects of corneal cross-linking on contrast sensitivity, visual acuity and corneal topography in patients with keratoconus. Cornea 2013; 32(5): 591-596. 